\title{
Neutron Protein Crystallography: A Unique Tool for Probing Enzyme Mechanism
}

\author{
Leighton Coates
}

Biology and Soft Matter Division, Oak Ridge National Laboratory, 1 Bethel Valley Road, Oak

Ridge, TN, 37831

Understanding how the structure and dynamics of an enzyme system governs function through the catalytic steps of an enzyme and binding of ligand remain a major challenge in molecular biophysics. For example, many biologically important reactions depend on the transfer of protons, and a complete understanding of the catalytic power of enzymes requires knowledge of the protonation status of residues throughout the reaction pathway. The uniqueness of neutrons is in their fundamental physical properties, which result in the strong scattering from the lightest atoms in the Periodic Table, and in the neutrons' benignity towards biological samples. Thus, whilst X-ray crystallography has unrivalled ability to determine the 'heavy' atom structure of biological systems, locating hydrogen atoms in proteins is challenging using $X$-ray techniques, and is complicated by radiation damage effects. The state-of-the-art in neutron macromolecular crystallography beamlines and perdeuteration of the sample for challenging crystal systems will be discussed along with several recent science examples. 